\title{
PROGRESS REPORT AND OPERATIONAL CONSIDERATION OF SUPERCONDUCTING RF CAVITY AT TLS
}

\author{
G.H. Luo, L.H. Chang, T.S. Hu ${ }^{+}$, M.C. Lin, and Ch. Wang \\ Synchrotron Radiation Research Center, Hsinchu 300, Taiwan, R.O.C. \\ ${ }^{+}$Physics Department, National Tsing-Hua University, Hsinchu, R.O.C.
}

\begin{abstract}
The Taiwan Light Source (TLS) is a third-generation light source with low operating energy, $1.5 \mathrm{GeV}$, which is used as ultra-violet and soft x-ray light source. A Superconducting RF cavity designed by Cornell University and tested at Cornell Electron Storage Ring (CESR) will be installed at TLS. The $500 \mathrm{~mA}$ beam current upgrade project at TLS is expected to reach the following basic goals: 1) reducing the longitudinal coupled bunch instability, 2) increasing the stored beam current by factor of two, 3) maintaining the beam lifetime by doubling the operating gap voltage. The RF cavity and beam parameters calculation will be discussed. Calculation for radiation shielding and cryogenic safety will be presented. The HFSS simulation results for the cavity modes, components of high-power waveguide transmission system and the coupling parameters will be addressed.
\end{abstract}

\section{INTRODUCTION}

There are three operational Doris cavities installed to provide electrons acceleration and energy compensation at TLS's booster and storage ring. Doris cavity functions and operates as expected in the booster ring to provide adequate acceleration with great reliability. However, the double cavity set with damping antenna attached to each cavity port did not provide sufficient damping mechanism for the generated Higher Order Modes (HOMs) at storage ring. A plunger-type tuner with precision controlled cavity body temperature has been implemented for each cavity. These efforts with RF voltage modulation keep the stability of photon spectrum within acceptable range for general synchrotron users.

It has been a strong demand to increase the photon flux by at least of factor two with the same lifetime and better photon stability from users' community. Evaluation of third Doris cavity, PEPII cavity, and SC cavity was carried out during 1998. The potential of operating SRF cavity up to $8 \mathrm{MV} / \mathrm{m}$ with $200 \mathrm{~kW}$ is very attractive to TLS. The SC cavity extends large flexibility for tuning the cavity to optimize the operation parameters.

The CESR's SC cavity has been chosen and contracted to ACCEL for manufacturing. The large beam duct on both side of the cavity will help the HOM being coupling out of the cavity without reducing the Q-value of the fundamental modes. A comparison of operation parameters for Doris cavity and CESR's SRF cavity is shown in Table 1.
The comparison of physical location between the operational Doris cavities and the future SC cavity is shown in Fig. 2. The down stream space of SC cavity is reserved for SC multipoles wiggler to generate $\mathrm{x}$-ray.

Table 1 . The comparison of operating parameters for the Doris cavities and SRF cavity.

\begin{tabular}{|c|c|c|}
\hline Parameter & Doris cavity & SRF cavity \\
\hline Beam Energy (GeV) & 1.5 & 1.5 \\
\hline Circumference (m) & 120 & 120 \\
\hline RF Frequency (MHz) & 499.666 & 499.666 \\
\hline Harmonic Number & 200 & 200 \\
\hline Beam Current (mA) & 200 & 500 \\
\hline Energy Spread & $0.075 \%$ & $0.075 \%$ \\
\hline Bunch Length (mm) & 9.2 & 6.5 \\
\hline \begin{tabular}{|l} 
Compaction Factor \\
\end{tabular} & 0.00678 & 0.00678 \\
\hline Energy Loss (keV/turn) & 128 & $128(168)$ \\
\hline RF Gap voltage $(\mathrm{kV})$ & 800 & 1600 \\
\hline Number of Cavities & 2 & 1 \\
\hline Number of klystrons & 2 & 1 \\
\hline Wall Dissip. (W/cavity) & $27.5 k$ & $<30$ \\
\hline Beam Power (kW) & 64 & $64(84)$ \\
\hline Klystron $P_{\text {out }}(\mathrm{kW} / \mathrm{kly})$. & 60 & $60(100)$ \\
\hline $\mathbf{R}_{\mathrm{s}} / \mathbf{Q}_{0}$ & 77.441 & 44.5 \\
\hline Synchro. Freq. (kHz) & 26.5 & 37.8 \\
\hline Energy Acceptance & $\pm 1.4 \%$ & $\pm 2.1 \%$ \\
\hline RF Transmission Line & EIA6 1/8" & WR1800 \\
\hline \begin{tabular}{|l|} 
Tuning Angle Offset \\
\end{tabular} & $0^{\circ}$ & $0^{0}>\psi_{\text {offset }}>-10^{\circ}$ \\
\hline
\end{tabular}

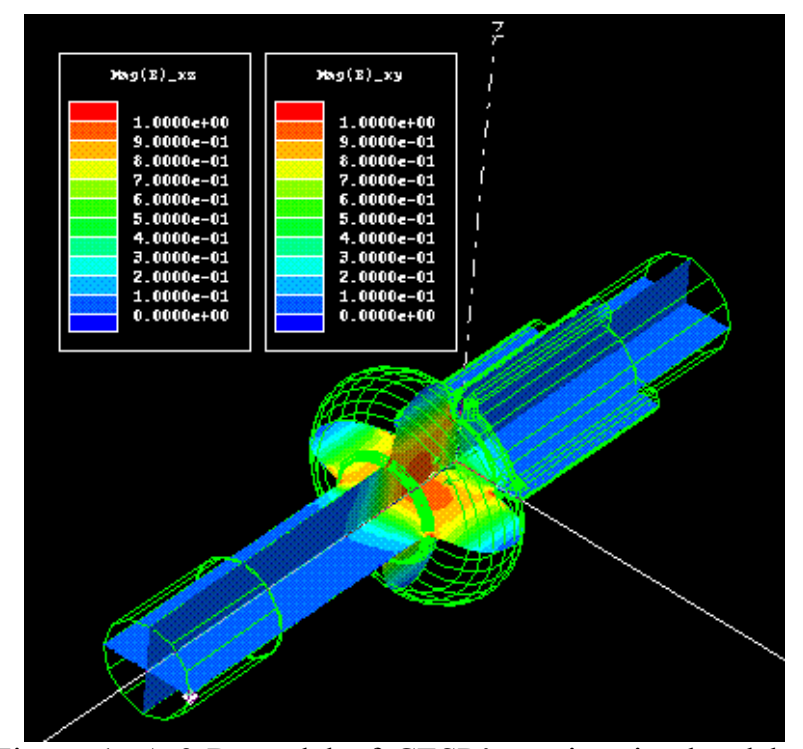

Figure 1. A 3-D model of CESR's cavity simulated by HFSS's Eigenmode Solver. 


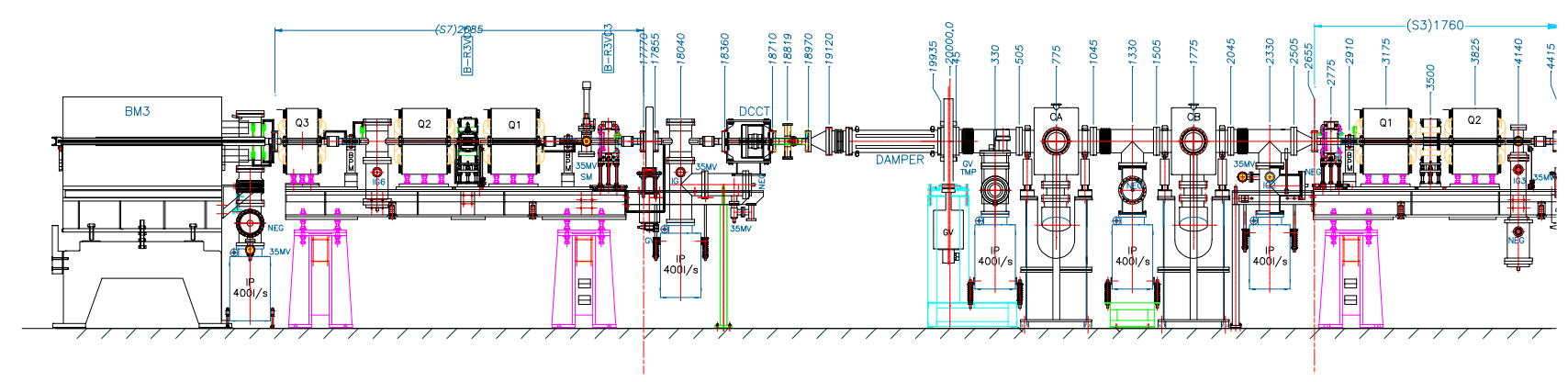

Figure 2a. Doris cavities and diagnostic instrumentation at TLS storage ring

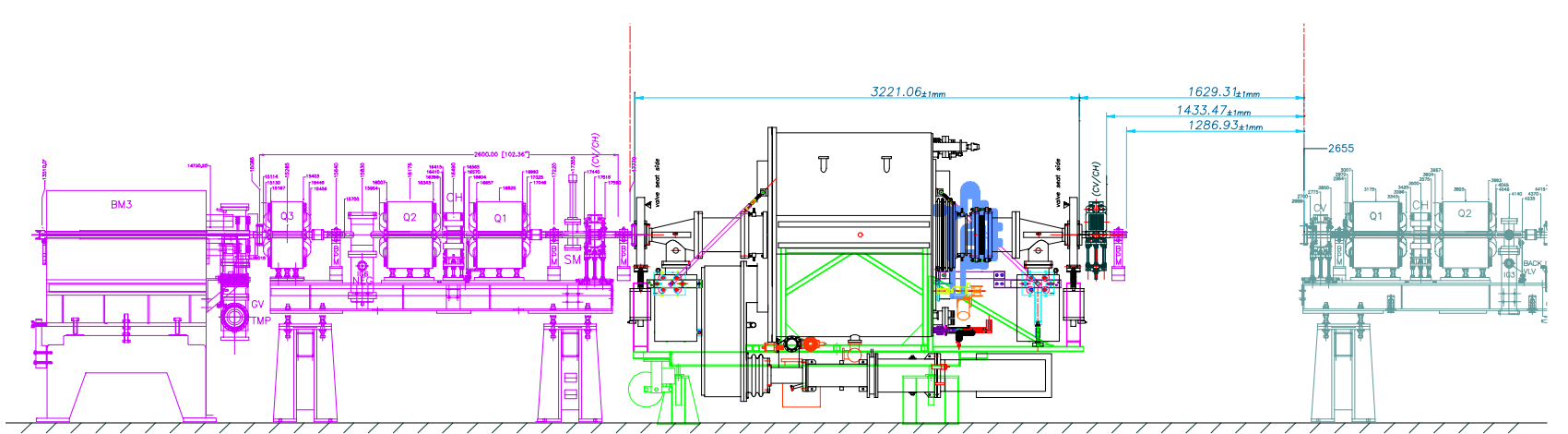

Figure $2 \mathrm{~b}$. Planned installation position and reserved space for SC multipoles wiggler

\section{NUMERICAL ANALYSIS OF THE CAVITY}

A commercial 3-D High-Frequency StructureSimulator (HFSS) [1] is used to simulate characteristics of the RF components. The $\mathrm{TM}_{010}$ resonance frequency, external Q, coupling coefficient $\beta$, HOMs, and the power handling capability of ceramic window are the main parameters that influence the operation of RF system and photon stability.

\subsection{The cavity modes, coupling coefficient and external $Q$}

The full structure of the cavity without input RF coupling slot has been simulated in order to find out the resonance frequency and shunt impedance of HOMs. Figure 1 shows the E-field intensity of fundamental, $\mathrm{TM}_{010}$, mode. Two ferrite sections were added to cavity in order to calculated $\mathrm{Q}_{0}$, and $\mathrm{R} / \mathrm{Q}$ value for the highly damped HOMs.

\subsection{RF windows and pump-out box taper design}

The 3-D model of matching rods, step changed waveguide and ceramic window was constructed to evaluate the matching condition of RF window section. The relative dielectric constant was found to be 9.6 with minimum reflection coefficient.

A sinusoid profile taper has been designed to provide a smooth transition between the cross section of RF window and the double elbow to reduce reflection coefficient. The cavity structure with input coupling section has been modeled to optimize the dimension of coupling tongue in order to find the proper coupling coefficient.

\section{SAFETY ISSUE}

To install the SC cavity into TLS's tunnel, two issues needs to be addressed and evaluated in advance to ensure the safety to environment, TLS staff and end users. The SC cavity can generate high-dosage radiation during high power processing due to high-energy multipacting and field-emission electrons. The SRF cryostat contains several hundreds liters of Liquid Helium (LHe) with running Liquid Nitrogen (LN2) to provide thermal isolation and keep adequate static heat-loss.

\subsection{Radiation Shielding}

The measurement of radiation dosage under different operation condition of SC cavity has been carried out at CESR. The data is taken during the processing period with measurement equipment 1 meter away from the cryostat [2]. Table 2 lists the dosage level and the operation condition. Table 3 lists the maximum annual dosage, which is regulated by TLS Safety Committee, for the staff who is working under radiation or non-radiation environment.

The shielding effects of high-energy photons is governed by following expression:

$$
\frac{d \Phi}{\Phi}=\mu d x \text { or } \Phi_{x}=\Phi_{0} e^{-\mu x}
$$


where $\Phi_{0}$ is the initial photon flux, and $\mu$ is the linear attenuation constant of shielding material. Combination of concrete and lead will be the materials used to shield the photons. According to the boundary value of Table 2 and 3 , the use factor of the cavity during the acceptance, the occupation factor of the SC cavity under normal operation, and the tenth value layer of the materials, the basic shielding wall will consist of $60 \mathrm{~cm}$ concrete wall surrounded the cryostat and $5 \mathrm{~cm}$ lead block installed perpendicular to the beam duct axis.

Table 2, Measured radiation dosage during high power RF processing [2]

\begin{tabular}{|c|c|}
\hline $\begin{array}{c}\text { RF acceleration gradient } \\
\mathrm{MV} / \mathrm{m}\end{array}$ & $\begin{array}{c}\text { Radiation level } \\
\mathrm{R} / \mathrm{hr}\end{array}$ \\
\hline $5(\mathrm{CW})$ & $\sim$ \\
\hline $6(\mathrm{CW})$ & 0.05 \\
\hline $7(\mathrm{CW})$ & 0.5 \\
\hline $8.5(\mathrm{CW})$ & 6 \\
\hline $11(\sim 2 \%$ duty cycle $)$ & 4.75 \\
\hline
\end{tabular}

Tabe 3, The annual dosage limitation of the TLS [3]

\begin{tabular}{|l|c|l|}
\hline $\begin{array}{l}\text { Type of working } \\
\text { Condition }\end{array}$ & $\begin{array}{l}\text { Max. Annual } \\
\text { Dosage (mRem) }\end{array}$ & \\
\hline Radiation & 5000 & $\begin{array}{l}\text { Atomic Energy } \\
\text { Council of ROC }\end{array}$ \\
\hline Non-radiation & 500 & $\begin{array}{l}\text { Safety Comm. } \\
\text { Of SRRC }\end{array}$ \\
Radiation & 200 & \\
Non-radiation & 20 & \\
Environment & 10 & \\
\hline
\end{tabular}

\subsection{Cryogenic System}

The full capacity of LHe vessel of the cryostat is 520 liters with operating pressure at 5 psig. The static heat loss of the cryostat is expected to be less than $35 \mathrm{~W}$ at 4.5 $\mathrm{K}$. There are two 1.5 " mechanical valves, one 2" burst disk connected to a 6 " venting tube into the atmosphere. The venting capability of the cryostat due to mishandling of the system can be evaluated by the follows with the assumption of flow rate below subsonic [4]:

$W=\frac{735 A K P_{1} F \sqrt{M}}{\sqrt{T Z}}$, where $F=\sqrt{\frac{k}{k-1}\left[\left(\frac{P_{2}}{P_{1}}\right)^{2 / k}-\left(\frac{P_{2}}{P_{1}}\right)^{(k+1) / k}\right]}$.

The $W$ is the gas flow rate in $[l b s / h r], A$ is the cross section in $\left[\mathrm{in}^{2}\right], T$ is the temperature ${ }^{o} R, Z$ is the compressible coefficient, $M$ is the molecular weight, $P_{1}$ is the inlet pressure in $[p s i a], k$ is the ratio of specific heat and $K$ is the valve's efficiency, $90 \%$ assumed. The setting values of mechanical relief valves are 8 psig and 10 psig, respectively. The 2" burst disk will break at 15 psig. Under these value, we can found the LHe can be evacuate within $30 \mathrm{sec}$ under the pressure of 2 atms.

The venting of LHe might be due to cavity quench, break of insulation vacuum, LN2 leak inside the cryostat, the crack of window and malfunction of refrigerator. For the case of cavity quench, a quench detector will activate to interrupt the input RF power and reduce RF loss on the cavity. A serious quench or heat inlet will trig the fast pressure sensor due to abrupt change of LHe vessel pressure. The RF input power, heater inside LHe vessel and the inlet LHe line will be shut off within millisecond.

The ceramic window serves as separation of vacuum and atmosphere. An additional gas $\mathrm{N}_{2}$ section, sealed with kepton and filled with 1 atm $\mathrm{N}_{2}$, is added as buffer to air. The leakage of ceramic window will bring in only gas $\mathrm{N}_{2}$ and the accompanied heat, $26.55 \mathrm{~kJ}$. There will be 10.2 liters LHe evaporated and create minute pressure increase.

The major catastrophe will be the heat inlet due to vacuum leak at cryostat or vacuum beam duct. There are experiments carried out at CERN and SSC $[5,6,7]$ to evaluate the pressure raise inside a cryostat causing huge heat inlet and rapid pressure change. From the experiments, the fixed leakage rate can be equivalent to a small orifice by the following expression [8]:

$$
Q=A P_{1} C^{\prime}\left(\frac{k T}{m} \frac{2 \gamma}{\gamma+1}\right)^{1 / 2}\left(\frac{2}{\gamma+1}\right)^{1 /(\gamma+1)} \text {, for } P_{2} / P_{1} \leq\left(\frac{2}{\gamma+1}\right)^{\gamma /(\gamma-1)}
$$

where $\gamma=1.4$ for air, $C^{\prime}=0.85$, and $A$ is the area of orifice. By the assumption, it implies that a small vacuum leakage hole, 0.1 " of radius, brings in several $\mathrm{kW}$ of heat intake to the cryostat. The design of the vent valves and venting piping is large enough to operate the system safely.

The maximum flow rate of LN2 for the cryostat and refrigeration system is 130 liters $/ \mathrm{hr}$ from a 20k liters storage tank. The oxygen concentration will change with the rate of $0.2 \%$ per hour at TLS experimental area, if LN2 spill into the experimental area at maximum flow rate. Other measure will be implemented at TLS to ensure the safety, e.g., the oxygen deficient monitors and sound alarms, portable compressed air capsule near the cryostat, link the RF input power with safety interlock.

\section{SUMMARY}

The RF structure and cavity models were setup to simulate the RF properties. A 3-D cryostat model for the analysis of magnetic shielding was under going by TOSCA and quasi-asymptotic analysis has accomplished [9]. The safety evaluation of cryostat has completed and presented to TLS Safety Committee. A turbine type cryogenic system with $450 \mathrm{~W}$ capacities at $4.5 \mathrm{~K}$ was contracted to Air-Liquide. Cavity vertical testing, HOM dampers and RF windows will be tested at CESR this summer. The cryostats are on the manufacturing batch at Meyer Tool.

\section{REFERENCES}

[1] HFSS, by Ansoft Corp., Pittsburgh, 1999

[2] H.Padamsee, S.Belomestnykh, private communication

[3] SRRC, Safety Guidelines, July 1995

[4] T.Flynn, Cryogenic Engineering, M. Dekker, 1997

[5] Ph. Leburn, CERN AT/95-30,

[6]H.Danielsson, ICEI Conference, 1992

[7] L.X. Jia, Advance in Cryo. Eng. Vol.43, 1998.

[8] J.F. O'Hanlon, $A$ User's Guide to Vacuum Technology, John Wiley \& Sons, 1989

[9] L.H. Chang, ISPMM, 2001 